\title{
Cuidados paliativos e conhecimento docente: diálogos possíveis na área da saúde
}

\author{
Palliative care and teacher knowledge: possible dialogues in the health area \\ Cuidados paliativos y conocimiento del profesorado: posibles diálogos en el área de la salud
}

Recebido: 12/12/2021 | Revisado: 16/12/2021 | Aceito: 24/12/2021 | Publicado: 04/01/2022

\author{
Erika Souza Garcia Ramos \\ ORCID: https://orcid.org/0000-0002-7201-0190 \\ Universidade de São Caetano do Sul, Brasil \\ E-mail: erika.ramos@online.uscs.edu.br \\ Rosamaria Rodrigues Garcia \\ ORCID: https://orcid.org/0000-0001-9454-6810 \\ Universidade de São Caetano do Sul, Brasil \\ E-mail: rosamaria.garcia@online.uscs.edu.br
}

\begin{abstract}
Resumo
O propósito deste artigo é apresentar o conhecimento e os sentimentos dos docentes na abordagem de temas relacionados aos cuidados paliativos como: finitude, morte e luto do curso de graduação em medicina da Universidade de São Caetano do Sul. Foi realizado um estudo transversal quantitativo e exploratório, realizado a partir dos preceitos e etapas do método científico, com aplicação de questionário semi-estruturado, por meio do googleforms aos professores do curso de graduação de medicina. O instrumento metodológico utilizado foi um questionário de 17 questões investigando informações pessoais, profissionais e sobre CP. A amostra foi composta por 106 participantes. A partir dos resultados, observamos que cerca de um terço dos docentes não têm formação na área de cuidados paliativos. Em consideração aos assuntos de morte e luto, menos de 50\% dos docentes tiveram esses conhecimentos na graduação. A pesquisa também revelou que aproximadamente $88 \%$ dos docentes conversam com os discentes sobre morte com segurança, em contraste com a literatura em que muitos médicos se sentem receosos ao tratar sobre morte e paliativismo, pelo fato de serem mal interpretados. Espera-se com a presente pesquisa que os resultados possam ser efetivamente conhecidos pelos docentes das faculdades de medicina, que atuem com mais sensibilidade às necessidades da população e indivíduo, e consequentemente compartilharem com os discentes uma abordagem mais humanizada.
\end{abstract}

Palavras-chave: Cuidados paliativos; Conhecimento docente; Atenção à saúde; Integralidade em saúde.

\begin{abstract}
The purpose of this article is to present the knowledge and feelings of professors in addressing themes related to palliative care such as: finitude, death and grief in the undergraduate medical course at the University of São Caetano do Sul. A quantitative and exploratory cross-sectional study was carried out, carried out from the precepts and stages of the scientific method, with the application of a semi-structured questionnaire, through Googleforms, to professors of the medical graduation course. The methodological instrument used was a 17-question questionnaire investigating personal, professional and PC information. The sample consisted of 106 participants. Based on the results, we observed that about a third of the professors do not have training in the area of palliative care. Considering the issues of death and mourning, less than 50\% of professors had this knowledge at graduation. The survey also revealed that approximately $88 \%$ of professors talk to students about death safely, in contrast to the literature in which many physicians feel fearful when dealing with death and palliative care, because they are misinterpreted. It is hoped with this research that the results can be effectively known by faculty of medicine, who act with more sensitivity to the needs of the population and the individual, and consequently share with students a more humanized approach.
\end{abstract}

Keywords: Palliative care; Teaching knowledge; Health care; Comprehensiveness in health.

\section{Resumen}

El propósito de este artículo es presentar los conocimientos y sentimientos de los profesores al abordar temas relacionados con los cuidados paliativos tales como: finitud, muerte y duelo en la carrera de medicina de la Universidad de São Caetano do Sul. Un análisis transversal cuantitativo y exploratorio. Se llevó a cabo el estudio., realizado a partir de los preceptos y etapas del método científico, con la aplicación de un cuestionario semiestructurado, a través de Googleforms, a los profesores del curso de graduación de Medicina. El instrumento metodológico utilizado fue un cuestionario de 17 preguntas que investigaba información personal, profesional y del PC. La muestra estuvo formada por 106 participantes. Con base en los resultados, observamos que alrededor de un tercio de los profesores no tiene formación en el área de cuidados paliativos. Teniendo en cuenta los problemas de la muerte y el duelo, menos del $50 \%$ de los profesores tenían este conocimiento al graduarse. La encuesta también 
reveló que aproximadamente el $85 \%$ de los profesores hablan con los estudiantes sobre la muerte de manera segura, en contraste con la literatura en la que muchos médicos sienten miedo al lidiar con la muerte y los cuidados paliativos, porque están mal interpretados. Con esta investigación se espera que los resultados puedan ser efectivamente conocidos por los profesores de medicina, quienes actúan con más sensibilidad a las necesidades de la población y del individuo, y consecuentemente comparten con los estudiantes un enfoque más humanizado.

Palabras clave: Cuidados paliativos; Enseñanza del conocimiento; Cuidado de la salud; Integralidad en salud.

\section{Introdução}

O envelhecimento populacional e os avanços da medicina moderna contribuíram para o aumento significativo da prevalência de doenças crônicas e degenerativas, e consequentemente na demanda por necessidades múltiplas de cuidado, entre elas os cuidados paliativos (Jackson, 2015). De acordo com a Organização Mundial de Saúde (OMS), essa realidade revela a importância da inclusão na formação médica de temas relacionados aos cuidados de fim de vida e o processo de morrer. (Castro, 2021).

A definição mais recente de cuidados paliativos (CP) foi publicada em 2018 pela International Association for Hospice \& Palliative Care (IAHPC):

cuidados holísticos ativos, ofertados a pessoas de todas as idades que se encontram em intenso sofrimento relacionado à sua saúde, proveniente de doença grave, especialmente aquelas que estão no final da vida. O objetivo do $\mathrm{CP}$ é, portanto, melhorar a qualidade de vida dos pacientes, de suas famílias e de seus cuidadores (IAHPC, 2018, p.1).

Para diminuir o sofrimento, Carvalho (2012) menciona ser necessário a identificação precoce, avaliação e tratamento da dor e outros problemas de dimensão física, psicológica, social e espiritual em um trabalho de equipe multiprofissional e interdisciplinar. Enfatizamos que todas essas dimensões não podem ser tratadas e abordadas por um único profissional. Por isso, as equipes de CP são multidisciplinares (ANCP, 2021).

Ao longo dos séculos, a intimidade do homem com a morte deu lugar a uma atitude de medo e negação, transformando-a em um tabu social (Hermes, 2013). Apesar da morte ser reconhecida por alguns como um processo natural, a maioria dos médicos, sejam eles com diferentes especializações e/ou com alta qualificação profissional, continua tendo dificuldades para lidar com a morte, relatando sentimentos de incômodo e angústia. O que se percebe é que a maioria deles lida com a morte no cotidiano da profissão, e com o passar do tempo, por meio de muito sofrimento, vai adquirindo maturidade profissional e emocional (Falcão, 2009).

A morte, continua Falcão (2009), é um fenômeno universal inerente à condição humana, permeada de simbolismos, significados e valores, variando no decorrer da história e entre as diversas culturas. O ser humano tem consciência da sua finitude, mas na maior parte do tempo ignora o assunto. Nossa sociedade vem cada vez mais tentando postergar esta hora, e para isso conta com a medicina e seus avanços científicos e tecnológicos (Kovács, 2005).

A formação médica, na sociedade contemporânea ocidental, é pautada no modelo cartesiano e técnico-científico de valorização da cura em detrimento do cuidado médico. No que diz respeito à cura, visa ao investimento na vida a qualquer preço, na qual a medicina de alta tecnologia se torna presente, e as práticas mais humanistas ficam em segundo plano. Já no paradigma do cuidar, Kovács (2012) aponta que há uma aceitação da morte como parte da condição humana, leva-se em conta a pessoa doente, e não somente a doença; enfatiza-se a multidimensionalidade da doença, a dor total.

Waldow (2009) menciona que são muitos os desafios enfrentados na educação básica dos profissionais de saúde, destacando entre eles: o despreparo dos próprios docentes em lidar com temas mais recentes e o avanço tecnológico que acentua a objetificação, a fragmentação e a padronização de experiências, anulando a subjetividade. Diante disso, pode-se perceber que cuidar no contexto atual é difícil, portanto educar para o cuidar também é desafiante. 
O conceito de saúde torna-se completo apenas quando tem seu sentido ampliado para além da questão biológica e abrange toda a esfera biopsicossocial e espiritual à qual o indivíduo se insere e se define. Essa complexidade deve ser sempre considerada quando se presta atendimento em saúde, seja este curativo ou paliativo. Devido a esta importância, surge a atual tendência de transformação das linhas curriculares formadoras de profissionais da saúde com a inserção de nuances de humanização (Oliveira; Ferreira \& Rezende, 2013).

A análise atenta dos currículos das escolas médicas mostra que estes privilegiam apenas o aprendizado das ferramentas de diagnóstico e de cura, e abordam nada ou quase nada sobre como cuidar de quem vai morrer. Além disso, aborda-se muito pouco com os estudantes sobre os sentimentos envolvidos no processo de morrer do ponto de vista do doente e da família, da mesma forma que não se diz ao aluno que o próprio profissional sente o impacto do sofrimento e da morte diários, geralmente sem ter como elaborar os sentimentos provocados em si mesmo pela dor de outro ser humano (Kovács, 2003).

De acordo com Mateus (2019), uma das grandes lacunas na formação acadêmica atual é a falta de discussão sobre a morte e o "paliativismo". O autor afirma que discutir temas como morte, finitude e "paliativismo" podem ajudar na consolidação da formação do indivíduo como humano, além de médico. Isso vai ao encontro do propósito dessa pesquisa.

Segundo Figueiredo e Stano (2013) as escolas de medicina beneficiariam a formação dos futuros médicos se incorporassem aos seus currículos o ensino de $\mathrm{CP}$, possibilitando complementar o aprendizado da medicina e proporcionando melhores recursos de cuidado na cura e na terminalidade de vida, bem como na assistência geral destinada ao paciente.

Tendo em vista essa discussão inicial, o objetivo desse artigo é compreender o conhecimento e os sentimentos dos docentes na abordagem de temas relacionados aos CP como: finitude, morte e luto do curso de graduação em medicina da Universidade Municipal de São Caetano do Sul (USCS).

\section{Metodologia}

Foi realizado um estudo transversal quantitativo e exploratório, realizado a partir dos preceitos e etapas do método científico, em que se busca respostas a problemas percebidos na sociedade, delimitados pelo pesquisador e que requerem investigação, sendo submetidos às etapas do método científico (Koche, 2011). Sobre os estudos de natureza exploratória: "pode-se dizer que estas pesquisas têm como objetivo principal o aprimoramento ou a descoberta de intuições. Na maioria dos casos, essas pesquisas envolvem: levantamento bibliográfico e entrevistas com pessoas que tiveram experiências práticas com o problema pesquisado" (Gil, 2002, p. 41).

Nesse sentido, a pesquisa envolveu um levantamento bibliográfica e aplicação de questionário semi-estruturado, por meio do googleforms aos professores do curso de graduação de medicina da Universidade Municipal de São Caetano do Sul (USCS), do campus Bela Vista em São Paulo.

Esta pesquisa foi aprovada pelo Comitê de Ética em Pesquisa da Universidade Municipal de São Caetano do Sul, sob parecer número 4.780.709 e CAAE 471132521600005510.

Foi utilizado o correio eletronico e aplicativo de mensagens para smartphone para envio de convite para participação na pesquisa, após leitura e concordância com o Termo de Consentimento Livre e Esclarecido (TCLE).

Foi realizado o cálculo amostral, considerando uma distribuição heterogênea da população, com intervalo de confiança de 95\% e erro amostral de 5\%, sendo obtido o resultado de 97 participantes para representatividade da amostra.

$\mathrm{O}$ instrumento metodológico de pesquisa, tinha duração de aproximadamente 15 minutos, composto por dezessete questões, sendo dezesseis objetivas e uma questão aberta, investigando informações pessoais, profissionais e sobre CP. 


\section{Resultados}

Foram enviados 129 convites individuais ao quadro total de docentes do curso de graduação em Medicina da Universidade Municipal de São Caetano do Sul, do campus Bela Vista, sendo duas tentativas de envio por e-mail, em sistema de cópia oculta, e duas tentativas por WhatsApp no grupo dos docentes; e após individualmente mais duas ou três tentativas aos que ainda não haviam respondido.

Após o envio do questionário a todos os professores, houve 111 (cento e onze) respostas, sendo 05 (cinco) replicadas que estavam exatamente iguais à primeira resposta, e portanto, foram excluídas, totalizando 106 participantes, obtendo-se a representatividade da amostra. O que se segue é uma análise referente ao perfil docente, conhecimentos e sentimentos na abordagem dos temas de CP.

Quanto aos dados demográficos doperfil docente, conforme a Tabela 1, a amostra foi composta por 55 professores do gênero masculino $(51,9 \%)$ e 51 do gênero feminino (48,1\%), idade média de 46,5 anos, $\pm 10,0$ DP, com mínima de 27 e máxima de 69 anos.

Considerando à formação acadêmica dos docentes, a maioria destes, 74 dos participantes possui graduação em medicina (70,0\%), seguidos por 08 fisioterapeutas (7,5\%), 07 enfermeiros (6,6\%), 04 dentistas (3,8\%), 03 biomédicos (2,8\%), 02 farmacêuticos (1,9\%), 02 biólogos (1,9\%), 02 psicólogos (1,9\%), 01 nutricionista ( $0,9 \%), 01$ fonoaudiólogo (0,9\%), 01 médico veterinário $(0,9 \%)$ e 01 advogado $(0,9 \%)$.

Com relação ao tempo de formado na graduação, a média foi de 21,9 anos, $\pm 9,6 \mathrm{DP}$, variando de 4 a 46 anos. Considerando a carreira acadêmica, em média o tempo de docência foi de 10,5 anos, $\pm 9,2 \mathrm{DP}$, variando de 1 a 42 anos. No que concerne à titulação acadêmica, 45 possuem doutorado (43,0\%), 35 têm mestrado (33,3\%), 17 possuem pós-graduação $(16,1 \%), 05$ possuem pós-doutorado $(4,8 \%)$ correspondendo a um biólogo, um dentista, um farmacêutico, um enfermeiro e um biomédico, 02 graduação $(1,9 \%)$ e 01 livre-docência $(0,9 \%)$.

No que diz respeito às metodologias ativas de ensino, a maioria dos participantes, 84 (79,25\%) possui formação, enquanto $22(20,75 \%)$ ainda não.

Tabela 1 - Perfil dos docentes.

\begin{tabular}{cc}
\hline Perfil dos docentes & Distribuição \\
\hline Gênero & \\
\hline Masculino & $55(51,9 \%)$ \\
Feminino & $51(48,1 \%)$ \\
\hline Formação & \\
\hline Médico & $74(70,0 \%)$ \\
Fisioterapeuta & $08(7,5 \%)$ \\
Enfermeiro & $07(6,6 \%)$ \\
Dentista & $04(3,8 \%)$ \\
Biomédico & $03(2,8 \%)$ \\
Farmacêutico & $02(1,9 \%)$ \\
Psicólogo & $02(1,9 \%)$ \\
Biólogo & $02(1,9 \%)$ \\
Nutricionista & $01(0,9 \%)$ \\
Fonoaudiólogo & $01(0,9 \%)$ \\
Veterinário & $01(0,9 \%)$ \\
Advogado & $01(0,9 \%)$ \\
\hline Titulação & \\
\hline Graduação & $02(1,9 \%)$ \\
\hline
\end{tabular}




\begin{tabular}{cc}
\hline Pós-graduação Lato Sensu & $17(16,1 \%)$ \\
Mestrado & $35(33,3 \%)$ \\
Doutorado & $45(43,0 \%)$ \\
Pós-doutorado & $5(4,8 \%)$ \\
Livre-docência & $1(0,9 \%)$ \\
\hline
\end{tabular}

Fonte: Dados de pesquisa (Ramos, 2021).

A finalidade do Gráfico 1 é apresentar o nível de formação em relação a temática de CP. Vale ressaltar que nessa questão o docente poderia assinalar mais de uma alternativa.

A partir das informações referentes ao gráfico 1, observamos que, considerando o tema de cuidado paliativo, houve predomínio de docentes (39 dos participantes, correspondendo a 36,8\%) que estudaram sozinhos, seguidos por 33 (31,1\%) que não possuem formação na área, $22(20,8 \%)$ que tiveram a temática na graduação, 22 que tiveram a temática na graduação (20,8\%), 22 afirmaram que tiveram na pós-graduação (20,8\%) e 17 fizeram curso específico (16,0\%). Já em relação à finitude, 36 não possuem formação (34,0\%), seguidos por 31 que estudaram sozinhos (29,2\%), 30 estudaram na graduação (28,3\%), 22 na pós-graduação $(20,8 \%)$ e 12 fizeram curso específico $(11,3 \%)$.

Considerando os assuntos morte e de luto, observou-se que (44\% e 39\% respectivamente) tiveram formação durante a graduação, todavia os participantes relataram que estes temas também foram contemplados individualmente (34\% em ambos).

Gráfico 1- Distribuição dos participantes de acordo com o nível de formação acadêmica sobre a temática de cuidados paliativos e afins.

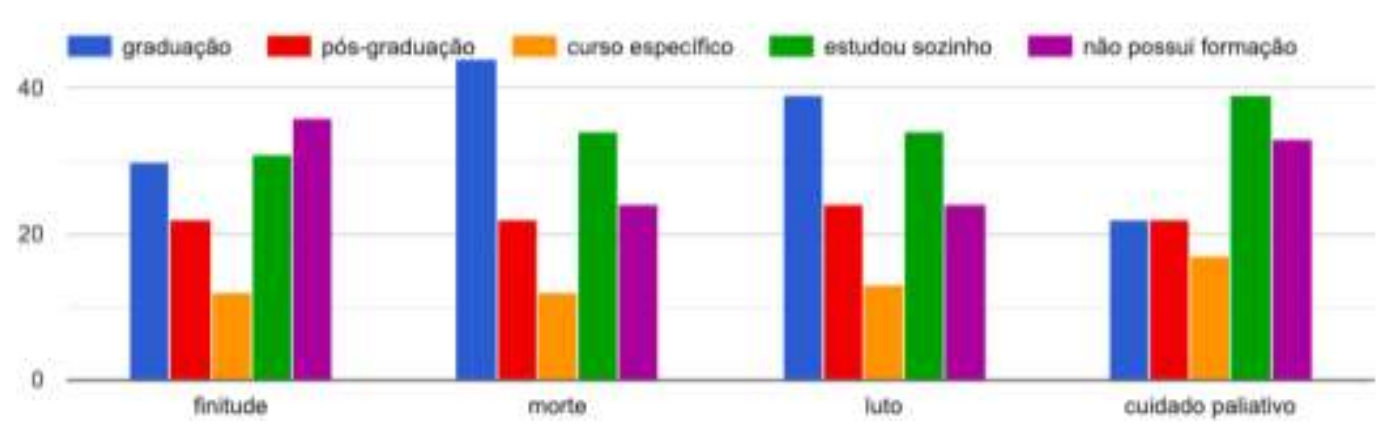

Fonte: Dados de pesquisa (Ramos, 2021).

O Gráfico 2 apresenta os sentimentos e percepção dos docentes quanto à abordagem dos temas. A partir das informações referentes ao Gráfico, percebe-se que, quanto ao tema morte, os docentes se sentem aptos, seguidos por parcialmente aptos (23\% e 25\% respectivamente). Enquanto que $19 \%$ se sentem parcialmente inaptos e $11 \%$ inaptos.

$\mathrm{Na}$ abordagem do tema luto, há um empate, em que $30 \%$ se consideram aptos e $30 \%$ parcialmente aptos. Da mesma forma, $31 \%$ se consideram parcialmente aptos e $24 \%$ parcialmente inaptos em abordar sobre finitude. Ressaltamos que, na abordagem do tema CP, observamos que $30 \%$ se consideram parcialmente inaptos e $29 \%$ parcialmente aptos. 


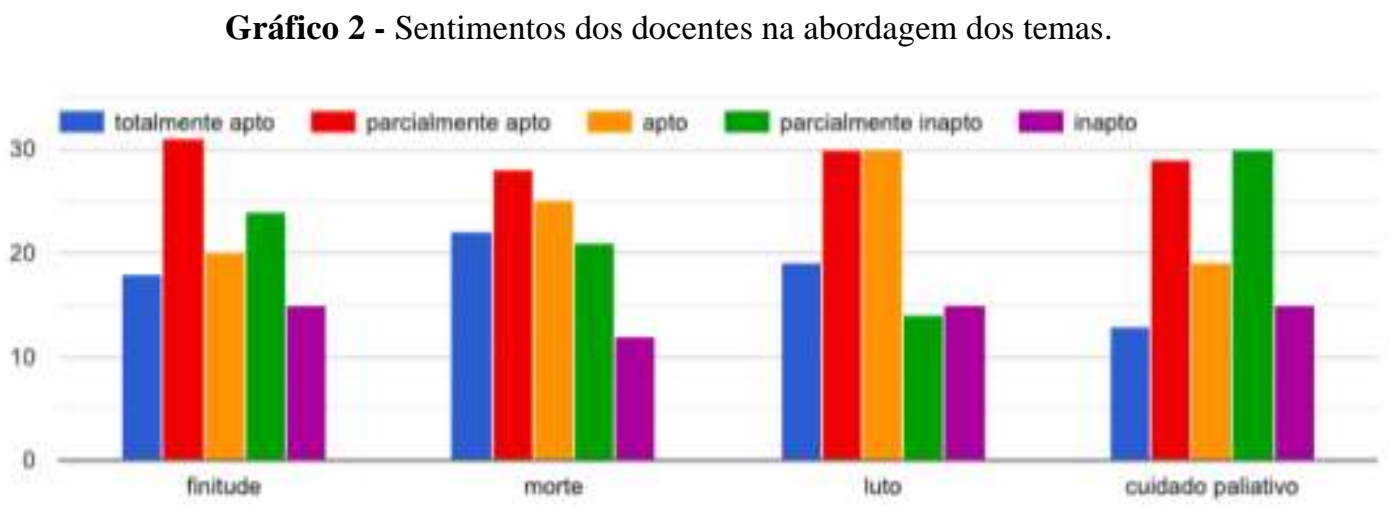

Fonte: Dados de pesquisa (Ramos, 2021).

É interessante observar que, quando questionamos sobre as reações e sentimentos perante a morte, apenas 51 docentes referiram sentir-se confortáveis (32,2\%), enquanto 06 referiram medo (3,8\%), 17 assinalaram insegurança (10,8\%), 11 angústia (7,0\%), 37 tristeza (23,4\%), 17 ansiedade (10,8\%), 12 despreparo (7,6\%), 07 frustração (4,4\%), dentre outros sentimentos, apresentados na tabela 2. Os sentimentos negativos perante a morte somaram $67,8 \%$, observando-se predominância em relação ao sentimento de conforto. Vale ressaltar que nessa questão o docente poderia assinalar mais de uma alternativa.

Tabela 2 - Reações e sentimentos dos docentes perante a morte.

\begin{tabular}{c|c}
\hline Reações e sentimentos & Distribuição \\
\hline Confortável & $(32,2 \%)$ \\
\hline Medo & $(3,8 \%)$ \\
Insegurança & $(10,8 \%)$ \\
Angústia & $(7,0 \%)$ \\
Tristeza & $(23,4 \%)$ \\
Despreparo & $(7,6 \%)$ \\
Ansiedade & $(10,8 \%)$ \\
Frustração & $(4,4 \%)$ \\
\hline
\end{tabular}

Fonte: Dados de pesquisa (Ramos, 2021).

O Gráfico 3 revela os temas abordados pelos docentes nas atividades acadêmicas das disciplinas ministradas, evidenciando-se morte (50\%), seguido por cuidado paliativo (43,4\%), luto (34\%) e finitude $(31,1 \%)$. Importante destacar que 32 docentes, o que corresponde a 30,2\% da amostra responderam que esta questão não se aplicava. 
Gráfico 3 - Temas abordados nas atividades acadêmicas das disciplinas ministradas.

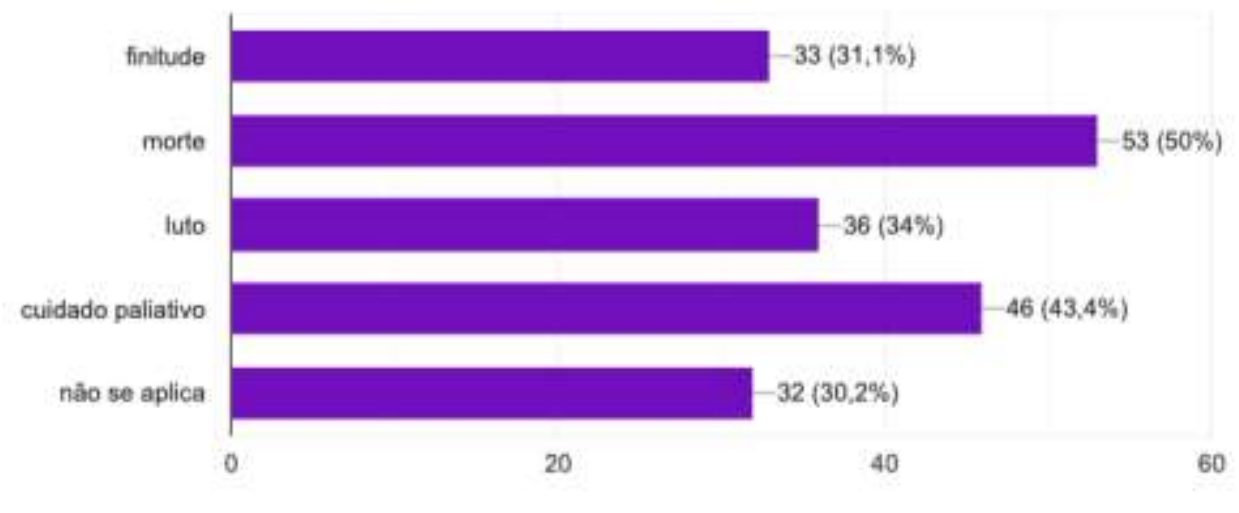

Fonte: Dados de pesquisa (Ramos, 2021).

O Gráfico 4 apresenta as estratégias de ensino utilizadas pelos docentes na abordagem dos temas. Foram observados os seguintes tipos: discussão de caso (53,8\%), situação problema $(50,0 \%)$, sala de aula invertida $(19,8 \%)$, aula expositiva (17\%), simulação (17,0 \%), team based lerning (TBL) (13,2\%), dramatização (11,3\%). Enquanto 19 docentes não abordam tais temas, correspondendo a $17,9 \%$ da amostra.

Os temas são abordados nas seguintes disciplinas: Saúde, doença e cuidado (SDC), estações clínicas (EC) e no internato de clínica médica. A disciplina SDC aborda necessidades de saúde por meio de situações-problema enquanto na EC há realização de histórias de vida, histórias clínicas e exame físico em pacientes simulados, relação médico paciente, raciocínio clínico-epidemiológico e planos terapêuticos frente à identificação de necessidades de saúde. O internato em clínica médica é um estágio intensivo de treinamento em serviço cujo objetivo central é a prática da conduta médica diante de pacientes de alta complexidade, internados em enfermarias de clínica médica e suas subespecialidades.

Gráfico 4 - Estratégias de ensino utilizadas nas abordagens dos temas relacionados aos cuidados paliativos

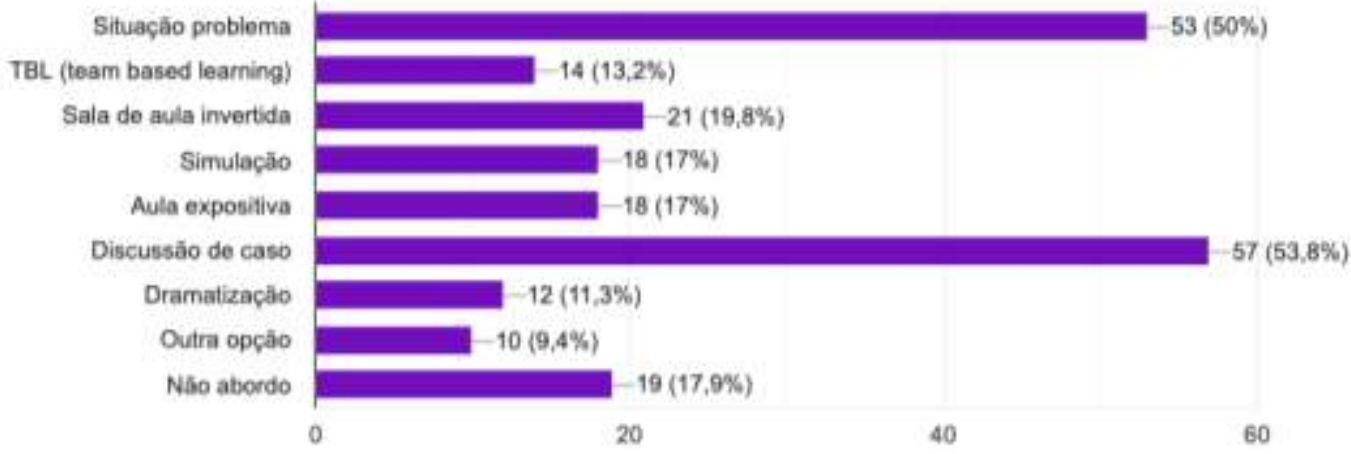

Fonte: Dados de pesquisa (Ramos, 2021).

O Gráfico 5 mostra a reação referida pelos docentes durante uma conversa sobre morte com o aluno. 
Gráfico 5 - Reação do docente durante conversa sobre morte com aluno

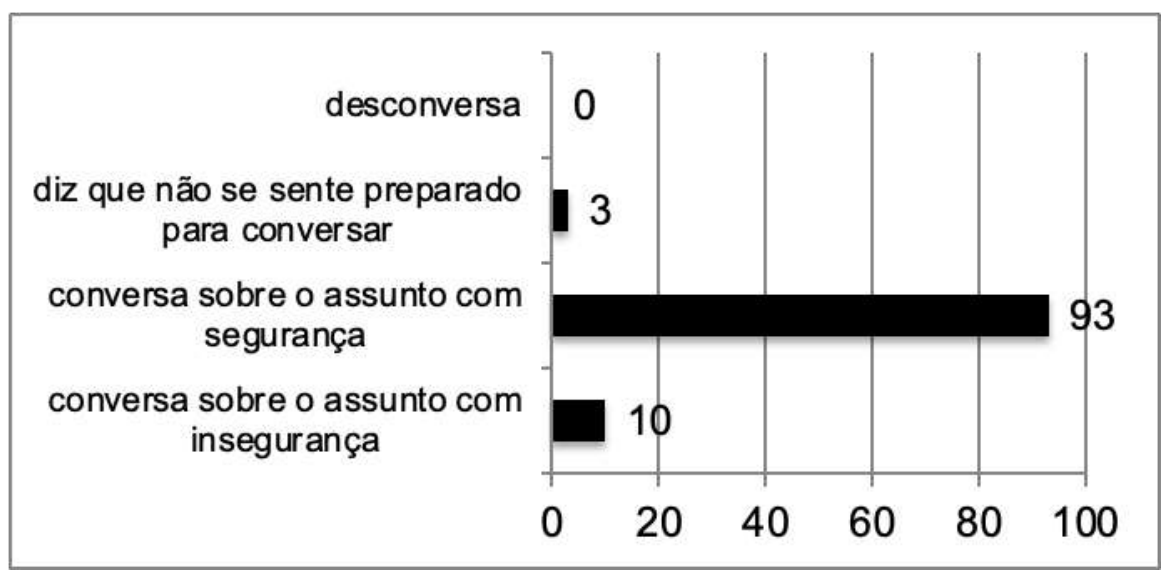

Fonte: Dados de pesquisa (Ramos, 2021).

Pode ser observado no gráfico 5, que 93 dos docentes conversam sobre o assunto com naturalidade $(87,7 \%)$, 10 conversam sobre o assunto com insegurança $(9,4 \%), 03(2,8 \%)$ não se sentem preparados para conversar $(2,8 \%)$, contudo nenhum dos docentes desconversa sobre o tema morte.

\section{Discussão}

Por meio dos resultados, observamos que cerca de um terço dos docentes não têm formação na área de CP. Esse dado também é sinalizado na pesquisa de Caldas (2018) em que o corpo docente possuia baixa capacitação nessa área do conhecimento, gerando um desafio na implementação do ensino de CP.

Em relação aos temas de morte e luto, menos de $50 \%$ dos docentes tiveram esses conhecimentos na graduação corroborando com Bifulco (2009) que evidencia principalmente por meio de "cursos", seguidos por "leitura bibliográfica", "palestra", "reportagem" e "vivência profissional" evidenciando a ausência do tema nos cursos de graduação. Embora parte da amostra tenha relatado que estudou sozinha a temática, não há indícios de incapacidade de abordar o assunto tendo em vista que os participantes referiram que se sentem aptos e parcialmente aptos. No entanto, não se anula a necessidade de realizarem uma formação mais sistematizada e formal.

O discente e o docente lidam com a morte de forma direta ou indiretamente, seja pela perda de um ente querido ou óbito de um paciente, respectivamente. Após a perda enfrentam o luto. Evidenciando a importância desses temas durante a graduação. É o que destaca Franco (2010) ao afirmar que é preciso entrar em contato a perda para aprender a lidar com as emoções e sentimentos para desenvolver competências e habilidades emocionais para atingir o envolvimento com o paciente com equilíbrio.

A pesquisa também revelou que aproximadamente $90 \%$ dos docentes conversa sobre morte com segurança com os discentes, em contraste, Mateus (2019) afirma que muitos médicos se sentem receosos ao tratar sobre morte e paliativismo, pelo fato de serem mal interpretados. Na pesquisa de Mateus foram analisados vivências de estágio de acadêmicos do décimo período de um curso de medicina de uma universidade do interior do Estado de Minas Gerais.

Vale ressaltar que no projeto político pedagógico da USCS encontramos CP e os temas relacionados em apenas duas atividades curriculares mas os alunos possuem oficina de luto na EC do primeiro ano, oficina de comunicação na EC do segundo ano e discutem indicações de CP no SDC do terceiro ano, configurando um continuum durante os primeiros anos da 
faculdade. Essas temáticas também poderiam estar presentes nas disciplinas de antropologia, práticas funcionais, saúde baseada em evidências, morfofuncional e internato de cirurgia. Além disso, sugere-se que essas temáticas possam ser incorporadas no projeto político pedagógico e mais vezes nas situações problemas discutidas no SDC ao longo dos quatro primeiros anos da faculdade.

Fonseca (2021) evidenciou o quanto é importante a abordagem sobre CP nas grades curriculares, sendo necessário a inclusão de disciplinas específicas e ações extracurriculares dos cursos da área da saúde.

Uma pesquisa da Unicamp acredita ser necessária a construção de um eixo formativo longitudinal que permeie os seis anos da formação médica, para abordar a formação da identidade profissional, a relação médico-paciente e os valores e virtudes relacionados à prática da medicina. Esse processo deve ser explicitado ao estudante para que ele se aproprie da própria formação (Scheweller, 2014). Levando em consideração que a construção do conhecimento é horizontal, o docente deve estar preparado independente da disciplina ou do ano da graduação para abordar tais assuntos.

Castro (2021), afirma ainda que, nos Estados Unidos os temas são ministrados por médicos de diversas especialidades, como geriatras, médicos de família e clínicos. No entanto, o autor aponta que são relatadas dificuldades em estratégias de ensino, ainda predominando táticas de leitura e discussão em pequenos grupos.

Enquanto na Universidade Municipal de São Caetano do Sul, os temas são trabalhados com clínicos, cirurgiões e outros profissionais da saúde. Além disso, possuem uma ampla variedade de estratégias de ensino: discussão de caso (principalmente), situação problema, sala de aula invertida, aula expositiva, simulação, team based lerning (TBL) e dramatização.

No atual estudo, a faixa etária dos profissionais interessados em participar de um curso de atualização na área de CP variam de 31 a 67 anos. Essa motivação parece decorrer da falta dos temas na graduação dos que têm muitos anos de formado bem como os que têm poucos anos configurando pouca mudança curricular nos últimos anos. Como a formação básica dos profissionais que lidam com pacientes que vivenciam o processo de morte não é suficiente, torna-se necessário o investimento na formação continuada sobre o assunto (Araújo, 2011).

A literatura sobre o conhecimento dos docentes a cerca dessas temáticas é escassa. O trabalho de Falcão (2009) evidencia que o tema morte permanece institucionalmente invisível, e se reforça justamente a ótica, criticada pelos docentes, da biomedicina, que desconhece ou desconsidera a integridade do paciente. Além de perceberem que a ausência de espaços institucionais de trocas entre si para o enfrentamento dos limites dos procedimentos técnicos, permanecem circunscritos em experiências individuais não compartilhadas.

Diante disso, propõem a criação de espaços institucionais, onde sistematicamente venha a ocorrer o compartilhamento de "ideias, experiências, sentimentos e expectativas" entre médicos docentes e estudantes (Falcão, 2009).

\section{Considerações Finais}

O objetivo principal deste artigo foi avaliar o conhecimento e os sentimentos dos docentes na abordagem de temas relacionados aos CP. Percebemos que se consideram com conhecimentos suficientes nessa área, um dado que confirmou nossa hipótese.

Por meio dos resultados, observamos que a maioria dos docentes, infelizmente, não percebem a importância de conhecer os temas relacionados aos CP para lidar com os discentes. Tendo em vista que é um tema que atravessa toda a futura prática clínica do médico.

Espera-se com a presente pesquisa que os resultados possam ser efetivamente conhecidos pelos docentes das faculdades de medicina, que atuem com mais sensibilidade às necessidades da população e indivíduo, e consequentemente compartilharem com os discentes uma abordagem mais humanizada. 
A limitação desse artigo se refere ao estudo realizado apenas em uma instituição, restringindo o universo de pesquisa. Como sugestão de trabalho futuro é o desenvolvimento de um curso de atualização com intuito de aquisição de conhecimentos e habilidades docentes, consequentemente com um melhor compartilhamento com os discentes. Destaca-se a necessidade de desdobramentos desta pesquisa, com objetivo de ampliar as estratégias dos docentes envolvidos e a compreensão dos gestores das faculdades de medicina quanto à importância do ensino de $\mathrm{CP}$ e temas relacionados.

\section{Agradecimentos}

Ao meu irmão Hugo que me apoiou, ajudou e revisou meu artigo. A minha querida orientadora e mestra, Rosamaria Rodrigues que esteve sempre presente e trouxe leveza para a nossa pesquisa, que me acolheu durante todo o percurso com paciência e carinho.

\section{Referências}

Academia Nacional de Cuidados Paliativos (ANCP). (2021). Manual de Cuidados Paliativos [Internet]. >https://paliativo.org.br/especialistas-em-medicinapaliativa>

Araujo, M. M. T. D. (2011). Comunicação em cuidados paliativos: proposta educacional para profissionais de saúde (Doctoral dissertation, Universidade de São Paulo).

Ariès, P. (1977). O homem diante da morte. Rio de Janeiro: Francisco Alves.

Bergmann, J. \& Sams, A. (2016) Sala de aula invertida: uma metodologia ativa de aprendizagem. Rio de Janeiro: LTC.

Bifulco, V. A. (2004). Princípios éticos e humanitários no cuidado de pacientes graves. Revista Meio de Cultura, 25, 19-20.

Bifulco, V. A., \& Iochida, L. C. (2009). A formação na graduação dos profissionais de saúde e a educação para o cuidado de pacientes fora de recursos terapêuticos de cura. Revista brasileira de educação médica, 33, 92-100. BOFF, L. Saber cuidar. Ética do humano - compaixão pela terra. $17^{\mathrm{a}}$ ed. Petrópolis, RJ: Vozes, 2011. 207p.

Bowlby, J., \& Cabral, A. (1990). Apego e perda: apego. In Apego e perda: apego (pp. xx-423).

Caldas, G. H. D. O., Moreira, S. D. N. T., \& Vilar, M. J. (2018). Cuidados paliativos: uma proposta para o ensino da graduação em Medicina. Revista Brasileira de Geriatria e Gerontologia, 21, 261-271.

Casellato, G. (2015). Luto não reconhecido: o fracasso da empatia nos tempos modernos. O resgate da empatia: suporte psicológico ao luto não reconhecido, $15-28$.

Cardinalli, I. E. (2011). Transtorno de estresse pós-traumático: um estudo fenomenológico-existencial da violência urbana. (Psychology thesis, Pontifícia Universidade Católica de São Paulo).

Carvalho, R. T., Souza, M. R. B., Franck, E. M., Polastrini, R. T. V., Crispim, D., Jales, S. M., ... \& Torres, S. H. B. (2018). Manual da residência de cuidados paliativos.

Carvalho, R. T.; Parsons, H. A. (Org.) Manual de Cuidados Paliativos ANCP 2.ed. São Paulo: s. n., 2012.

Polla, C. C. (2006). Nada a caminho: Impessoalidade, niilismo e técnica na obra de Martin Heidegger. Cadernos de Filosofia Alemã: Crítica e Modernidade, (10), 131-140.

Castro, A. A., Taquette, S. R., \& Marques, N. I. (2021). Cuidados paliativos: inserção do ensino nas escolas médicas do Brasil. Revista Brasileira de Educação Médica, 45.

dos Santos Fonseca, L., de Araújo, M. S., Santos, R. N., da Silva Sá, L. T. G., de Oliveira Santos, J. C., de Lima Ferreira, L. L., ... \& Kameo, S. Y. (2021). Cuidados paliativos: Conhecimento de acadêmicos da saúde. Research, Society and Development, 10(6).

Elsner, F., Centeno, C., Cetto, G., Conno, F., Ellershaw, J., Eychmuller, S., ... \& Mason, S. (2013). Recommendations of the European Association for Palliative Care (EAPC) for the development of undergraduate curricula in palliative medicine at European medical schools.

Falcão, E. B. M., \& Mendonça, S. B. (2009). Formação médica, ciência e atendimento ao paciente que morre: uma herança em questão. Revista Brasileira de Educação Médica, 33, 364-373.

Figueiredo, M. D. G. M. C. D. A., \& Stano, R. D. C. M. (2013). O estudo da morte e dos cuidados paliativos: uma experiência didática no currículo de medicina. Revista Brasileira de Educação Médica, 37, 298-306.

Figueiredo, M. T. (2003). Educação em cuidados paliativos: uma experiência brasileira. Mundo saúde (Impr.), 165-170.

Fonseca, J. P. D. (2004). Luto antecipatório. In Luto antecipatorio (pp. 183-183). 
Franco, M. H. P., \& Conselho Regional de Medicina do Estado de São Paulo. (2008). Luto em cuidados paliativos. Conselho Regional de Medicina do Estado de São Paulo (Org.). Cuidado paliativo, 559-570.

Gil, A. C. (2002). Como elaborar projetos de pesquisa (Vol. 4, p. 175). São Paulo: Atlas.

Heidegger, M. (1990). Ser e Tempo. Tradução revisada de Marcia Sá Schuback. Petrópolis: Vozes.

Hermes, H. R., \& Lamarca, I. C. A. (2013). Palliative care: an approach based on the professional health categories. Ciencia \& saude coletiva, 18(9), 25772588 .

IAHPC. Global Consensus based palliative care definition. (2018).Houston, TX: The International Association for Hospice and Palliative Care. https://hospicecare.com/what-we-do/projects/consensus-based-definition-of-palliative-care/definition/

Jackson, K. (2015). Palliative Care and the Global Goal for Health. World Hospice and Palliative Care Association, International Association for Hospice and Palliative Care, and International Children's Palliative Care Network. Accessed January, 6.

Koche, J. C. (2011). Fundamentos de metodologia científica: teoria da ciência e iniciação à pesquisa. Petrópolis, RJ : Vozes.

Kovács, M. J. (2003). Educação para a morte: desafio na formação de profissionais de saúde e educação. In Educação para a morte: desafio na formação de profissionais de saúde e educação (pp. 175-175).

Kovács, M. J. (2010). Sofrimento da equipe de saúde no contexto hospitalar: cuidando do cuidador profissional. O mundo da saúde, 34(4), 420-429.

Kovacs, M. J. (2005). Educação para a morte: sugestões de linhas de ações para o psicólogo. São Paulo: Editora Casa do Psicólogo.

Kübler, R. E. (1996). Sobre a morte e o morrer: o que os doentes têm para ensinar a médicos, enfermeiros, religiosos e aos próprios parentes.

Magalhães, M. V., \& de Assunção Melo, S. C. (2015). Morte e luto: o sofrimento do profissional da saúde. Psicologia e Saúde em debate, 1(1), 65-77.

World Health Organization. (2002). National cancer control programmes: policies and managerial guidelines. World Health Organization.

Nestel, D., \& Tierney, T. (2007). Role-play for medical students learning about communication: guidelines for maximising benefits. BMC medical education, 7(1), 1-9.

Oliveira, J. R. D., Ferreira, A. C., \& Rezende, N. A. D. (2013). Ensino de bioética e cuidados paliativos nas escolas médicas do Brasil. Revista Brasileira de educação médica, 37(2), 285-290.

Parkes, C. M. (1998). Luto estudos sobre a perda na vida adulta (Vol. 56). Summus editorial.

Pessini, L., \& Bertachini, L. (2004). Humanização e cuidados paliativos. Edições Loyola.

Resolução n. ${ }^{\circ}$, de 23 de jun. de 2014. Institui Diretrizes Curriculares Nacionais do curso de graduação em medicina. Diário Oficial da União. Brasília

Santos, A. F. J., Ferreira, E. A. L., \& Guirro, U. B. P. (2020). Atlas dos cuidados paliativos no Brasil 2019.

Steinert, Y., Mann, K., Centeno, A., Dolmans, D., Spencer, J., Gelula, M., \& Prideaux, D. (2006). A systematic review of faculty development initiatives designed to improve teaching effectiveness in medical education: BEME Guide No. 8. Medical teacher, 28(6), 497-526.

Stroebe, M., Schut, H., \& Boerner, K. (2010). Continuing bonds in adaptation to bereavement: Toward theoretical integration. Clinical psychology review, 30(2), 259-268.

Schweller, M., Wamserlei, J., Strazzacappa, M., Sá, F. C., Celeri, E. H. R. V., \& Carvalho-Filho, M. A. (2014). Metodologias ativas para o ensino de empatia na graduação em medicina: uma experiência da Unicamp. Cadernos ABEM, 10, 36-46.

Toledo, A. P. D., \& Priolli, D. G. (2012). Cuidados no fim da vida: o ensino médico no Brasil. Revista Brasileira de Educação Médica, 36, $109-117$.

Universidade Municipal de São Caetano do Sul. Projeto Político Pedagógico do Curso de Medicina. São Paulo: 2016.

Waldow, V. R. (2009). Reflexões sobre Educação em Enfermagem: ênfase em um ensino centrado no cuidado. Mundo Saúde, 33(2), 182-8.

Worden, J. W. (1998). Terapia do luto: Um manual para profissionais da Saúde Mental. Porto Alegre: Artes Médicas. 